\title{
Integral Adaptive Control And Single Parameter Identification Based On Terminal Attractor And Sigmoid Function
}

\author{
Zexue $\mathrm{Li}^{1,}$ a , Jun Peng ${ }^{2, b}$, Junwei Lei ${ }^{3, c}$ \\ ${ }^{1,3}$ Department of control engineering, Naval aeronautical and astronautical University, Yantai, China \\ ${ }^{2}$ Receiving and Training Center of New Equipments, Naval Aeronautical and Astronautical \\ University, Yantai, China \\ alizexue1024@126.com, ${ }^{b}$ pengjun1024@126.com
}

Keywords: adaptive control, parameter identification, one order system

\begin{abstract}
In order to achieve adaptive control and single parameter identification of one order system, an ordinary adaptive control method is used in this paper. Integral control, Terminal attractor and Sigmoid function is combined to achieve parameter identification. Based on Laypunov method, an adaptive control law is designed to make the system insensitive to parameter uncertainties. In the end, a conclusion can be made that theoretical analysis is correct and parameter identification method is effective by numerical simulation.
\end{abstract}

\section{Introduction}

Adaptive control has been researched more and more in recent years by scientists in every country because of its strong ability to cope uncertain parameters in system model ${ }^{[1-6]}$. As the demand for control performance increases, the problem of time-variance parameters of controlled system is more and more important. Terminal control has robustness and is not sensitive to parameter variation. Sigmoid function is one of the most transfer function in artificial neural networks, it is used in artificial neural networks ${ }^{[7-9]}$ at the earliest, it has continuity, smooth, differentiability, boundedness. In this paper, an improved integral adaptive control that is based on Terminal attractor and Sigmoid function is proposed, the simulation results show that system parameters can be identified.

\section{Problem Description}

One order system can be written as:

$$
\dot{x}=a x+u
$$

where $a$ is unknown constant parameter, the goal is designing a controller such that the system state $x$ can trace the expected value $x^{d}$.

\section{Design Adaptive Identification Controller}

An ordinary adaptive control method is used as follows, define a error variable as $z_{1}=x_{1}-x_{1}^{d}$, then

$$
\dot{z}_{1}=\dot{x}_{1}-\dot{x}_{1}^{d}=a x+u
$$

Design state feedback control law as:

$$
u=-\hat{a} x-\sum_{i=1}^{n} k_{i} f_{i}\left(z_{1}\right)-k_{s 1} \int z_{1} d t
$$

Choose $n=5, k_{i}>0$

$$
f_{1}\left(z_{1}\right)=z_{1}, \quad f_{2}\left(z_{1}\right)=z_{1}^{3}, \quad f_{3}\left(z_{1}\right)=z_{1}^{1 / 3}
$$




$$
\begin{array}{r}
f_{4}\left(z_{1}\right)=\frac{z_{1}}{\left|z_{1}\right|+\varepsilon}, \quad \varepsilon=0.2, \\
f_{5}\left(z_{1}\right)=\frac{1-e^{-\tau z_{1}}}{1+e^{-\tau z_{1}}}, \quad \tau=0.5
\end{array}
$$

where $f_{3}\left(z_{1}\right)$ is Terminal attractor, and $f_{5}\left(z_{1}\right)$ is Sigmoid function, $f_{4}\left(z_{1}\right)$ and $f_{5}\left(z_{1}\right)$ both have boundedness, Obviously, $f_{i}\left(z_{1}\right)$ meet $z_{1} f_{i}\left(z_{1}\right) \geq 0$, then

$$
\dot{z}_{1}=\tilde{a} x-\sum_{i=1}^{n} k_{i} f_{i}\left(z_{1}\right)-k_{s 1} \int z_{1} d t
$$

where the error variable $\tilde{a}$ can be defined as:

$$
\tilde{a}=a-\hat{a},
$$

design regulating law:

$$
\dot{\hat{a}}=\Gamma z_{1} x
$$

where $\hat{a}$ is unknown estimated parameter value, choose initial value $\hat{a}(0)=0$, then

$$
\dot{\tilde{a}}=-\dot{\hat{a}}
$$

choose Lyapunov function:

$$
V=\frac{1}{2} z_{1}^{2}+\frac{1}{2 \Gamma} \tilde{a}^{2}+\frac{k_{s 1}}{2}\left(\int z_{1} d t\right)^{2}
$$

Then

$$
\dot{V}=z_{1} \dot{z}_{1}+\frac{1}{\Gamma} \tilde{a} \dot{\tilde{a}}+k_{s 1} z_{1} \int z_{1} d t
$$

Then:

$$
\dot{V}=z_{1} \tilde{a} x-\sum_{i=1}^{n} k_{i} z_{1} f_{i}\left(z_{1}\right)-\frac{1}{\Gamma} \tilde{a} \Gamma z_{1} x=-\sum_{i=1}^{n} k_{i} z_{1} f_{i}\left(z_{1}\right) \leq 0
$$

So $z_{1} \rightarrow 0$.

\section{Parameter Identification Result Analysis}

When $z_{1} \rightarrow 0$, where $u=-\hat{a} x$, then

$$
\dot{z}_{1}=a x-\hat{a} x+k_{s 1} \int z_{1} d t=\tilde{a} x+k_{s 1} \int z_{1} d t
$$

When $z_{1} \rightarrow 0$, there is $\dot{z}_{1} \rightarrow 0$, then there is $\dot{z}_{1}=\tilde{a} x+k_{s 1} \int z_{1} d t=0$, so the parameter can be identified.

Obviously, if there is an integral adaptive control, the parameter can be identified.

\section{Numerical Simulation}

Choose $a=3, x_{1}^{d}=2, x_{1}(0)=-1$, use Simulink in Matlab, the program can be written as: 


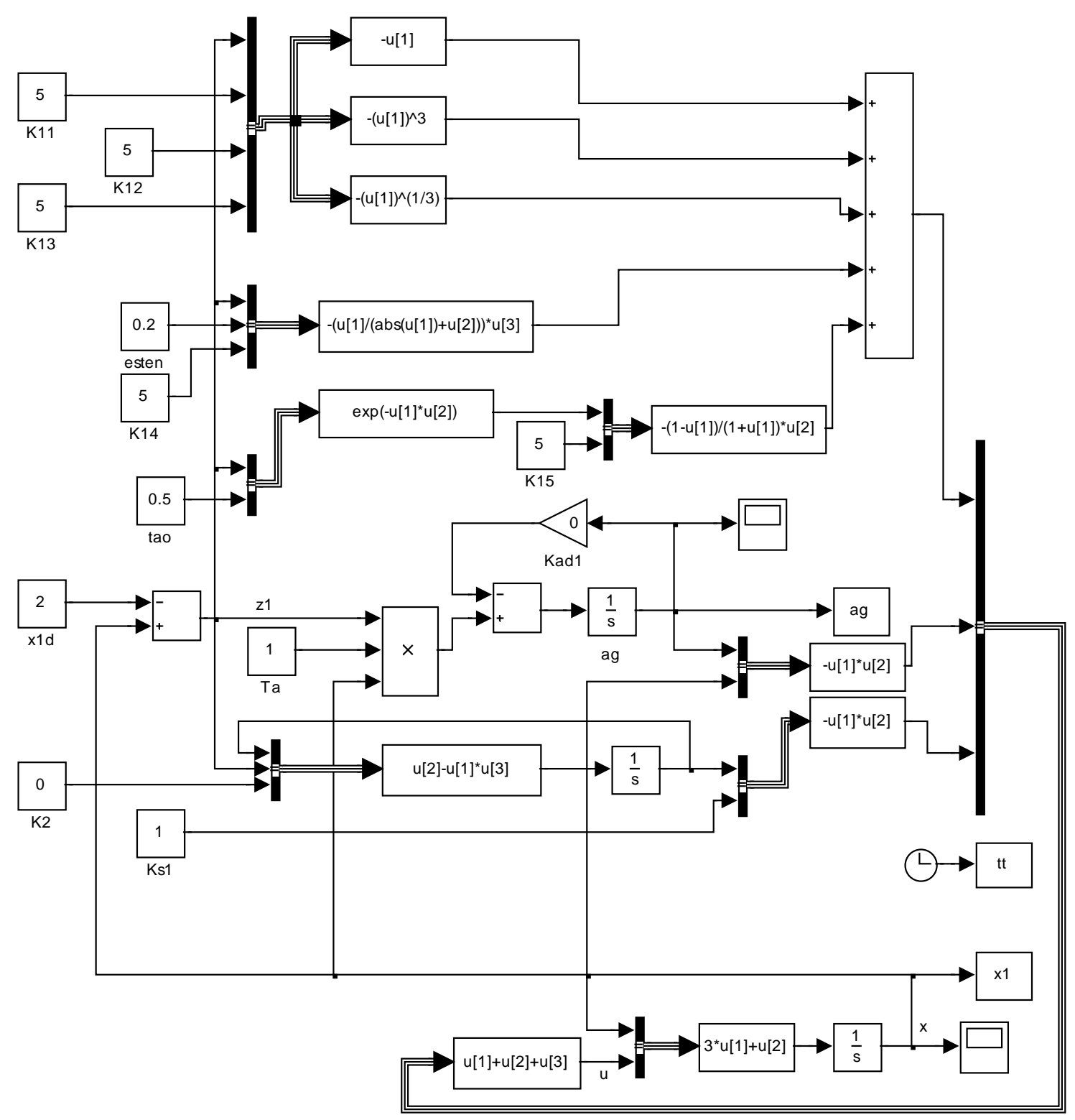

Fig.1 Program

Choose $k_{1}=5, k_{s 1}=1, \Gamma_{a}=1, k_{2}=k_{3}=k_{4}=k_{5}=5, k_{a d 1}=0, \tau=0.5, \varepsilon=0.2$, the simulation results are as follows:

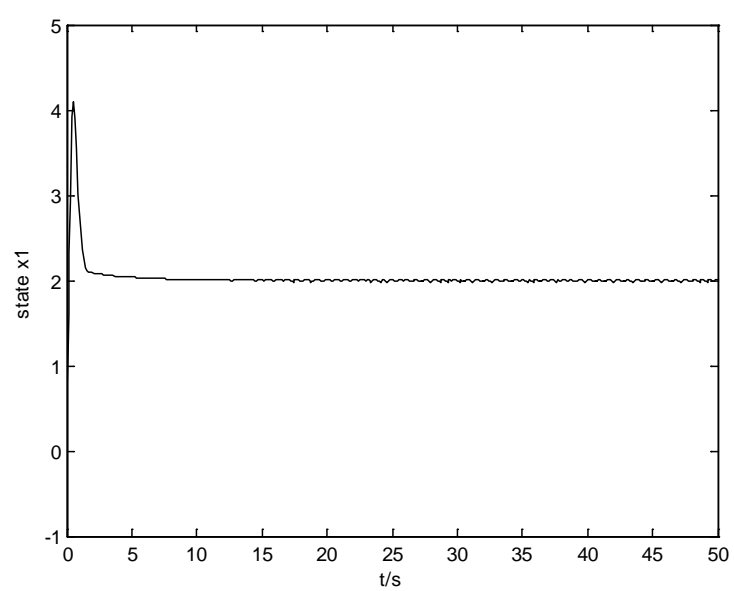

Fig. 2 state $x$

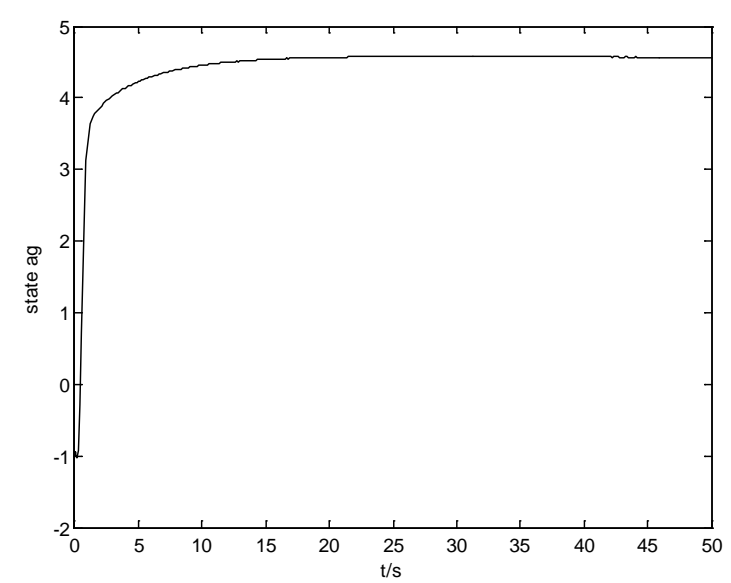

fig.3 state a

The simulation results show that the error of system is small, but the error of parameter 
identification is large, the main reason is that there is a nonlinear function.

Consider decreasing system shock, that is $k_{4}=0.2, \varepsilon=2$, the simulation results are as follows:

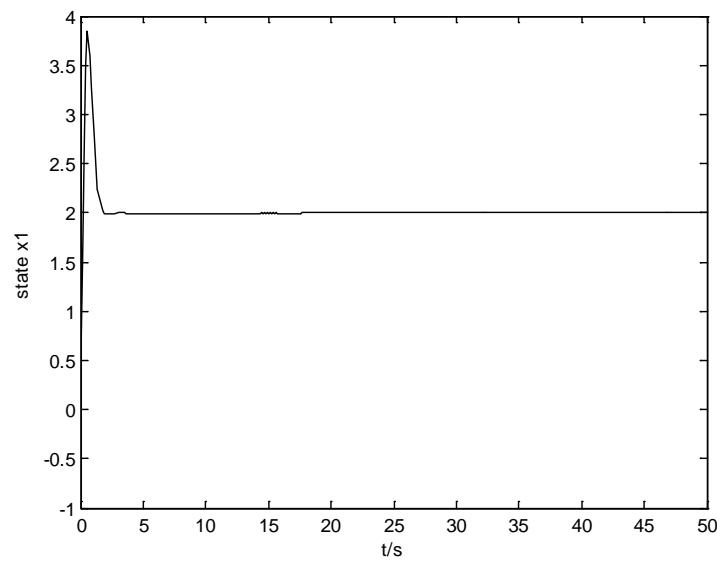

Fig.4 state $\mathrm{x}$

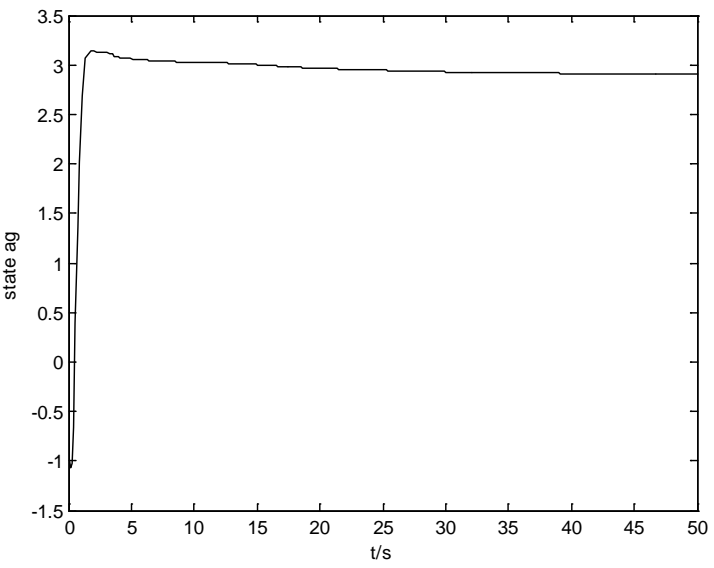

fig.5 state a

The simulation results show that the error of system is small, and the error of parameter identification is small. This one of advantage of nonlinear function, but the disadvantage is to use variable step emulation. Because Terminal function and Sigmoid function is used, it make the effect of integral decrease. So effect to the error of parameter identification decrease, integral of error as follows:

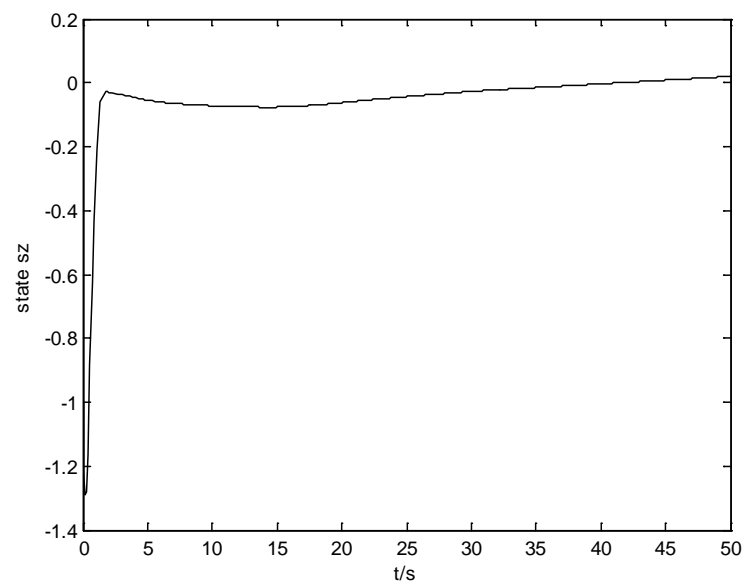

Fig.6 integral of error

\section{Conclusion}

Terminal function and Sigmoid function can decrease control parameter, it make the effect of integral decrease, and it make steady state error decrease. But the disadvantage is that variable step emulation must be used, because fixed step can not use nonlinear function's the characteristic of variable gain.

\section{Reference}

[1] Carrasco R C, Forcada M L. Simple strategies to encode tree automata in sigmoid recursive neural networks[J]. IEEE Transactions on Knowledge and Data Engineering 2001, 13(2): 148-156.

[2] Basterretxea K, Tarela J M. Del Campo I. Implementation issue of sigmoid function and its derivative for VLSI digital neural networks[J]. Electronics Letters, 2002, 38(1).

[3] Basterretxea K, Tarela J M. Del Campo I. Approximation of sigmoid function and derivative for hardware implementation of artificial neurons[j]. IEEE Proceedings online, 2004, 151(1). 
[4] David K. Schmidt.Modeling and Near-Space Station-Keeping Control of a Large High-Altitude Airship.Journal of Control and Dynamics,2007,30(2):540 547

[5] David K. Schmidt,James Stevens,Jason Roney.Near-Space Station-Keeping Performance of a Large High-Altitude Notional Airship.Journal of Control and Dynamics,2007,44(2):611 615

[6] Donald J.McTavish,Kyle Davidson.Practical Large-Motion Modeling of Geometrically Complex Flexible Vehicles:A Consistent-Mass Standard-FEM Based,All Terms Included Formulation.47th AIAA/ASME/ASCE/AHS/ASC Structures,Structural Dynamics, and Materials Conference.2006-1664

[7] Duc Cuong Quach, Shuang Huang, Quan Yin, Chunjie Zhou. An improved Direct Adaptive Fuzzy controller for an uncertain DC Motor Speed Control System. TElkomnika, indonesian journal of electrical engineering. February 2013; Vol 11, No 2: 1083-1092.

[8] Yonghong Zhu, Qing Feng, Jianhong Wang. Neural Network-based Adaptive Passive Output Feedback Control for MIMO Uncertain System. TElkomnika, indonesian journal of electrical engineering. October 2012; Vol 10, No 6: 1263-1272.

[9] Wen Xinling, Chen Yu. Research of the Nonlinear System Identification Based on the Volterra RLS Adaptive Filter Algorithm. TElkomnika, indonesian journal of electrical engineering. May 2013; Vol 11, No 5: 2277-2283. 